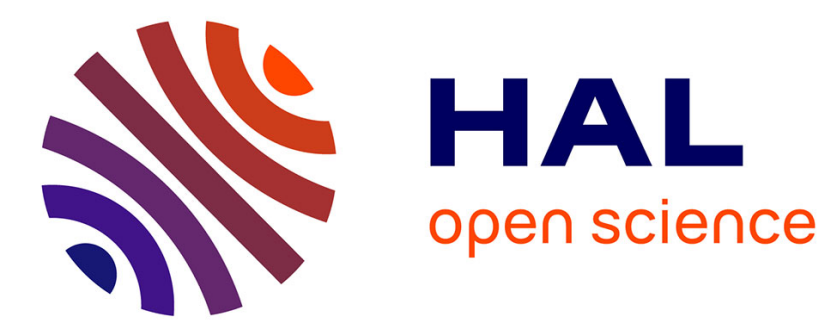

\title{
Cooperative-cum-Constrained Maximum Likelihood Algorithm for UWB-based Localization in Wireless BANs
}

Gia-Minh Hoang, Matthieu Gautier, Antoine Courtay

\section{To cite this version:}

Gia-Minh Hoang, Matthieu Gautier, Antoine Courtay. Cooperative-cum-Constrained Maximum Likelihood Algorithm for UWB-based Localization in Wireless BANs. IEEE International Conference on Communications (ICC15), Jun 2015, London, United Kingdom. hal-01175913

\section{HAL Id: hal-01175913 \\ https://inria.hal.science/hal-01175913}

Submitted on 13 Jul 2015

HAL is a multi-disciplinary open access archive for the deposit and dissemination of scientific research documents, whether they are published or not. The documents may come from teaching and research institutions in France or abroad, or from public or private research centers.
L'archive ouverte pluridisciplinaire HAL, est destinée au dépôt et à la diffusion de documents scientifiques de niveau recherche, publiés ou non, émanant des établissements d'enseignement et de recherche français ou étrangers, des laboratoires publics ou privés. 


\title{
Cooperative-cum-Constrained Maximum Likelihood Algorithm for UWB-based Localization in Wireless BANs
}

\author{
Gia-Minh HOANG, Matthieu GAUTIER, and Antoine COURTAY \\ University of Rennes 1, IRISA, INRIA, France \\ gia-minh.hoang@etudiant.univ-rennes1.fr, \{matthieu.gautier, antoine.courtay\}@irisa.com
}

\begin{abstract}
Wireless Body Area Network (BAN) is a mainstream technology for numerous application fields (medicine, security, sport science...) and precise determination of wireless sensors' positions responses to the great needs in many applications. In addition, Ultra Wide Band (UWB) radio is an attractive technology to achieve the centimeter-level distance measurements. However, the aggregation of the distance information remains a challenge and this paper presents a cutting-edge method for performing the accurate localization in wireless BAN. To this aim, by fully exploiting its unique features, a novel Cooperative-cumConstrained Maximum Likelihood (CCML) localization algorithm is developed. Simulation results and UWB-based platform validation show absolute agreement with theoretical prediction and improvement over previous studies by Hamie et al and Mekonnen et al.
\end{abstract}

\section{INTRODUCTION}

Wireless Body Area Network (BAN) is a short-range Wireless Sensor Network (WSN) composed of small, low-power, wearable or implanted electronic sensors on, around or inside the human body. It supports the data communication over short distances with the other sensors or with the data center for many special purposes. Thus, various potential mobile, personal and body-centric applications are promised to fulfill the market needs in short term. In most applications, the localization of the sensors is required and the accuracy plays a key role. In the context of BAN, the requirement of accuracy is highly demanding that many conventional localization systems such as well-known GPS fail to satisfy or do not work. Thus, a great deal of time and efforts have gone into investigating new localization systems that take into account the other BAN constraints such as low energy consumption, low cost, low computation, and wearable.

In general WSNs and specific BANs, the unknown location of a node (say the target node) is determined in two steps [1]. The first one is measurement step. In this first step, a target node exchanges packets with a few neighboring nodes whose positions are known a priori (say the reference nodes). From these communications, one or more position-related metrics are extracted [1][2] and the relative distances between each pair of nodes can be inferred from these metric. By employing trilateration technique [1][3], the second step called position estimation step aggregates these measurements as input of a position estimator or algorithm to compute the target node's position in a particular predefined coordinate system as output.
Due to critical accuracy requirements, technologies and algorithms must be dedicated to wireless BAN. In [3], by exploiting the very high time resolution of Ultra Wide Band (UWB) radio, the UWB nodes that measure time-based metrics such as Time of Arrival (ToA) can estimate very precisely their relative distances. Furthermore, low-power and low-cost implementation of Impulse Radio UWB (IR-UWB) communication systems meets the key requirements for wearable sensors. These aspects make UWB an attractive technology to improve the measurement step in the BAN context. However, when being associated with classical position estimation techniques, this high accuracy can be partially or completely vanished by the indoor propagation conditions or is not enough for very high accuracy requirements. To tackle these issues, the geometrical constraints imposed by the human body can be used to yield performance gain [4][5]. Inspired by the results in [4][5], in this paper, we aim to perform the UWBbased localization in BAN by proposing a novel Maximum Likelihood (ML) algorithm which accounts for the unique characteristics of BAN. Although this has been studied in the literature (i.e. [4][5]), our work considers several impractical assumptions such as 2D localization in [4] and unrealistic disposition of the body-strapped sensors in both [4] and [5]. Besides, the accuracy can be improved by cooperation between wireless nodes [2][6]. Thus, our proposal includes new body constraints and enables cooperative localization which have not been exploited to the fullest in previous efforts. This proposal is theoretically evaluated in terms of accuracy, complexity and empirically validated using real IR-UWB platforms.

The paper is structured as follows. In Section II, we provide an overview of dedicated localization systems for wireless BAN and state the location estimation problem. Section III focuses on our proposed CCML localization algorithm. Next, Section IV accounts for the evaluation framework including the simulation setup and performance results. Real experiments are addressed in Section $\mathrm{V}$ in order to evaluate the algorithm performance with real measurements. Finally, Section VI draws general conclusions.

\section{System Setup AND PROblem Formulation}

This section introduces the typical UWB-based localization system for BAN. It can be classified in two categories: rela- 
tive localization and absolute localization [1][2][4][5]. While relative localization refers to the system where the reference nodes are attached onto the body to form a Local Coordinate System (LCS), absolute localization has the reference nodes installed at fixed positions outside the body to define a Global Coordinate System (GCS).

In this paper, only relative localization is addressed. The set of reference nodes is strapped directly into the moving body at some special positions that are independent of the body mobility or gestures (e.g. usually on the torso) to defines a LCS on the human body. Therefore, the positions of the body mounted nodes can be known a priori. Figure 1 illustrates a typical relative localization scenario. The reference nodes are placed on known positions that are not influenced by the body attitude or mobility such as the chest, the back, and the hip. The number of reference devices should not be less than 3 for 2D positioning and 4 for 3D case without any prior knowledge about the target nodes' positions. Besides, the best placement of reference nodes should be tetrahedral [1], hence, the 4 reference sensors are arranged as follows: one on the chest, one on the back, the two remaining sensors on the hip. For the disposition of the target devices, we avoid attaching these on the joints/bends due to sensors' possible displacement. Instead, the disposition of target nodes relies on the Xsens MVN system [7].

Notations: Throughout this paper, we will use the following notation. $\left\{\boldsymbol{T}_{\boldsymbol{i}}\right\}_{i=1 \ldots N_{t}}$ represents the unknown positions of the $N_{t}$ target sensors, $\left\{\boldsymbol{R}_{\boldsymbol{i}}\right\}_{i=1 \ldots N_{r}}$ indicates the known a priori positions of the $N_{r}$ reference devices in the LCS on the torso and $\left\{\boldsymbol{J}_{\boldsymbol{i}}\right\}_{i=1,4,7,10}$ denotes 4 special body joints/bends whose positions with respect to the LCS are also known a priori (e.g. the shoulder and the hip joints) as illustrated in Figure 1. Intuitively, the positions of the nodes as well as the body joints can be represented in vector form as $\boldsymbol{R}_{\boldsymbol{i}}=\left[R_{i}^{(x)}, R_{i}^{(y)}, R_{i}^{(z)}\right]^{T}, \boldsymbol{T}_{\boldsymbol{i}}=$

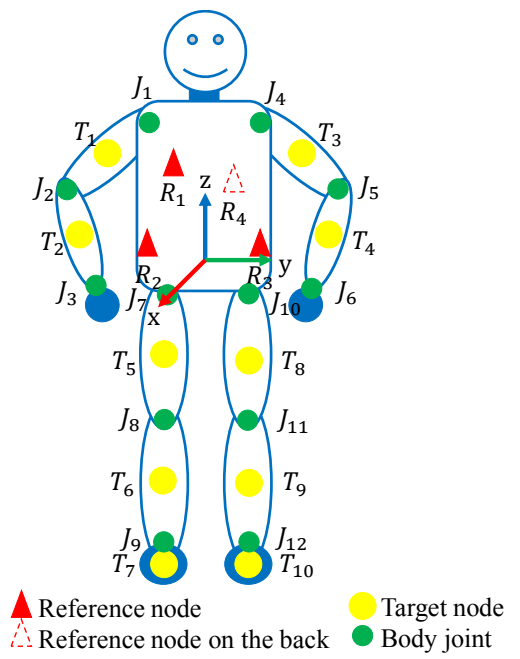

Figure 1. Relative localization system for BAN. The reference (red) nodes are attached on the torso while the target (yellow) nodes are mounted on the limbs to capture the motion.
$\left[T_{i}^{(x)}, T_{i}^{(y)}, T_{i}^{(z)}\right]^{T}$, and $\boldsymbol{J}_{\boldsymbol{i}}=\left[J_{i}^{(x)}, J_{i}^{(y)}, J_{i}^{(z)}\right]^{T}$. Let $\boldsymbol{\theta}=$ $\left[T_{1}^{(x)}, T_{1}^{(y)}, T_{1}^{(z)}, \ldots, T_{N_{t}}^{(x)}, T_{N_{t}}^{(y)}, T_{N_{t}}^{(z)}\right]^{T}$ be the estimate vector, which consists of unknown parameters. In the same way, we denote the collection of peer-to-peer ranging measurements by the vector $\tilde{\boldsymbol{d}}=\left[\tilde{d}_{11}, \tilde{d}_{12}, \tilde{d}_{13}, \ldots, \tilde{d}_{1 N_{t}}, \tilde{d}_{21}, \ldots, \tilde{d}_{N_{r} N_{t}}\right]^{T}$, where $\tilde{d}_{i j}$ is the ranging measurement between reference node $i$ and target node $j$. So an important point to remember is that $d_{i j} \neq d_{j i}, \forall i \neq j$.

As soon as these ranging measurements are determined by the IR-UWB ToA information as proposed in [3], localization algorithms estimate the positions of the target sensors in the LCS. Briefly, given the positions $\left\{\boldsymbol{R}_{\boldsymbol{i}}\right\}_{i=1 \ldots N_{r}}$, some special joints' positions (i.e. $\left\{\boldsymbol{J}_{\boldsymbol{i}}\right\}_{i=1,4,7,10}$ as in Figure 1) in the LCS and the ranging information $\tilde{\boldsymbol{d}}$, the objective is to estimate the vector $\boldsymbol{\theta}$.

\section{LOCAliZATION Algorithms}

\section{A. Conventional ML Localization}

The ML estimator pays attention to the statistics of the noise sources and maximizes the following likelihood function [1][4]:

$$
p(\tilde{\boldsymbol{d}} \mid \boldsymbol{\theta})=\prod_{i=1}^{N_{r}} \prod_{j=1}^{N_{t}} p\left(\tilde{d}_{i j} \mid \boldsymbol{\theta}\right)
$$

where $p(\cdot \mid \boldsymbol{\theta})$ denotes the conditional probability density function given parameter $\boldsymbol{\theta}$. And the ML estimator is as follows:

$$
\hat{\boldsymbol{\theta}}=\arg \max _{\boldsymbol{\theta}} \prod_{i=1}^{N_{r}} \prod_{j=1}^{N_{t}} p\left(\tilde{d}_{i j} \mid \boldsymbol{\theta}\right) \text {. }
$$

Let consider a simple scenario where the ranging errors are modeled as centered independent Gaussian variables i.e.

$$
\tilde{d}_{i j} \sim \mathcal{N}\left(d_{i j}, \sigma_{i j}^{2}\right)
$$

where $d_{i j}=\left\|\overrightarrow{R_{i} T_{j}}\right\|$ denotes the real distance between the reference node $i$ and the target node $j$, then the likelihood function of $\boldsymbol{\theta}$ takes the form:

$$
\begin{aligned}
p(\tilde{\boldsymbol{d}} \mid \boldsymbol{\theta}) & =\prod_{i=1}^{N_{r}} \prod_{j=1}^{N_{t}} \frac{1}{\sqrt{2 \pi \sigma_{i j}^{2}}} \exp \left[-\frac{f(\boldsymbol{\theta}, \tilde{\boldsymbol{d}})}{2 \sigma_{i j}^{2}}\right], \\
f(\boldsymbol{\theta}, \tilde{\boldsymbol{d}}) & =\left(\tilde{d}_{i j}-\sqrt{\left(\boldsymbol{R}_{\boldsymbol{i}}-\boldsymbol{T}_{\boldsymbol{j}}\right)^{T}\left(\boldsymbol{R}_{\boldsymbol{i}}-\boldsymbol{T}_{\boldsymbol{j}}\right)}\right)^{2} .
\end{aligned}
$$

As a result, the ML has now the formula of the non-linear Weighted Least Squares (WLS) estimator which yields:

$$
\hat{\boldsymbol{\theta}}=\arg \min _{\boldsymbol{\theta}} \sum_{i=1}^{N_{r}} \sum_{j=1}^{N_{t}} w_{i j} f(\boldsymbol{\theta}, \tilde{\boldsymbol{d}}),
$$

where $w_{i j} \triangleq 1 / \sigma_{i j}^{2}$ plays the role of weight which reflects the accuracy and the reliability of the measurement $\tilde{d}_{i j}$. Accordingly, when the measurements have different uncertainties, unreliable ones are down-weighted in the likelihood function 
and vice versa, trusted ones are over-weighted. The vector of unknown parameter $\boldsymbol{\theta}$ in the expression (6) cannot be described in close-form solution. Numerical methods such as Nelder-Mead method [8] are employed to solve this non-linear problem instead.

\section{B. Improved ML Localization}

In this part, we propose to adapt the standard ML estimator into the context of BAN.

1) Constrained ML Algorithm: To increase the accuracy, several constraints imposed by the body can provide the ML estimator with extra information. For example, the target node $T_{1}$ maintains a fixed distance to the shoulder joint $J_{1}$ as shown in Figure 2. This constrained localization has been already addressed in [4][5], however, our new constraints are more realistic because of considering both 3D localization and practical placement of the sensors on the body which avoids attaching sensor on the body joints/bends. Without loss of generality, only two sensors $T_{1}$ and $T_{2}$ on the left arm (see Figure 2) are analyzed due to the equivalent roles of the sensors on the arms and legs.

Assuming that all the ranging measurements are independent and equivalent (i.e. $\sigma_{i j}=$ const, $\forall i, j$ ), the formula (6) with $N_{t}=2$ for the two nodes $T_{1}, T_{2}$ and $N_{r}=4$ becomes:

$$
\hat{\boldsymbol{\theta}}=\arg \min _{\boldsymbol{\theta}} \sum_{i=1}^{4} \sum_{j=1}^{2}\left(\tilde{d}_{i j}-\sqrt{\left(\boldsymbol{R}_{\boldsymbol{i}}-\boldsymbol{T}_{\boldsymbol{j}}\right)^{T}\left(\boldsymbol{R}_{\boldsymbol{i}}-\boldsymbol{T}_{\boldsymbol{j}}\right)}\right)^{2},
$$

where $\boldsymbol{\theta}=\left[\boldsymbol{T}_{\mathbf{1}}, \boldsymbol{T}_{\mathbf{2}}\right]^{T}=\left[T_{1}^{(x)}, T_{1}^{(y)}, T_{1}^{(z)}, T_{2}^{(x)}, T_{2}^{(y)}, T_{2}^{(z)}\right]^{T}$ is the estimate vector.

Mathematically, the constraints imposed by the left arm can be formulated as follows:

$$
\begin{aligned}
& \boldsymbol{c}_{\boldsymbol{e q}}\left(\boldsymbol{\theta}, \boldsymbol{J}_{\mathbf{1}}, \boldsymbol{J}_{\mathbf{2}}\right)=\left[\begin{array}{c}
c_{e q 1}\left(\boldsymbol{\theta}, \boldsymbol{J}_{\mathbf{1}}\right) \\
c_{e q 2}\left(\boldsymbol{\theta}, \boldsymbol{J}_{\mathbf{2}}\right)
\end{array}\right] \\
& =\left[\begin{array}{c}
\sqrt{\left(\boldsymbol{T}_{\mathbf{1}}-\boldsymbol{J}_{\mathbf{1}}\right)^{T}\left(\boldsymbol{T}_{\mathbf{1}}-\boldsymbol{J}_{\mathbf{1}}\right)}-\left\|\overrightarrow{T_{1} J_{1}}\right\| \\
\sqrt{\left(\boldsymbol{T}_{\mathbf{2}}-\boldsymbol{J}_{\mathbf{2}}\right)^{T}\left(\boldsymbol{T}_{\mathbf{2}}-\boldsymbol{J}_{\mathbf{2}}\right)}-\left\|\overrightarrow{T_{2} J_{2}}\right\|
\end{array}\right]=\mathbf{0},
\end{aligned}
$$

where $\boldsymbol{c}_{\boldsymbol{e} q}\left(\boldsymbol{\theta}, \boldsymbol{J}_{\mathbf{1}}, \boldsymbol{J}_{\mathbf{2}}\right)$ is the matrix composed of the equality constraints of the target nodes which are determined by their positions on the body.

Note that the position of the left shoulder joint $J_{1}$ and two fixed distance $\left\|\overrightarrow{T_{1} J_{1}}\right\|,\left\|\overrightarrow{T_{2} J_{2}}\right\|$ are known a priori whereas the position of the left elbow joint $J_{2}$ is still unknown. Under

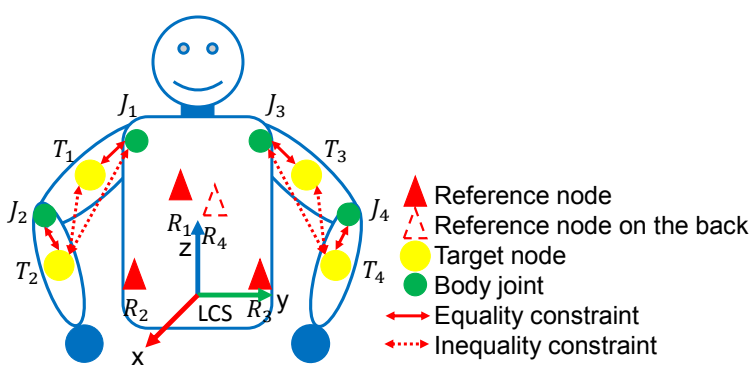

Figure 2. Constraints in BAN. normal circumstances, when relying on the placement of target node in Xsens MVN system [7], we have to sacrifice the second constraint (i.e. $c_{e q 2}\left(\boldsymbol{\theta}, \boldsymbol{J}_{\mathbf{2}}\right)=0$ ). Therefore, our main contribution in the constrained algorithm is to gain back this constraint. By using analytic geometry, the position of the left elbow $J_{2}$ can be referred by one of the left shoulder joint $J_{1}$ and one of the target node $T_{1}$ as follows:

$$
\boldsymbol{J}_{\mathbf{2}}=\boldsymbol{J}_{\mathbf{1}}+\frac{\left\|\overrightarrow{J_{2} J_{1}}\right\|}{\left\|\overrightarrow{T_{1} J_{1}}\right\|}\left(\boldsymbol{T}_{\mathbf{1}}-\boldsymbol{J}_{\mathbf{1}}\right)
$$

Substituting $\boldsymbol{J}_{\mathbf{2}}$ in (8) by (9), the constraints now are independent of the position of $J_{2}$. Moreover, we propose to use also another type of constraint i.e. inequality constraints which have not been investigated in previous studies. For example, in Figure 2, the target node $T_{2}$ cannot reach positions that are 50 centimeters away from the corresponding shoulder joint $J_{1}$. However, stricter inequality constraints can be computed by using the following triangle inequalities:

$$
\begin{aligned}
& \boldsymbol{c}_{\boldsymbol{i n e q}}\left(\boldsymbol{\theta}, \boldsymbol{J}_{\mathbf{1}}, \boldsymbol{J}_{\mathbf{2}}\right)=\left[\begin{array}{c}
c_{\text {ineq } 1}\left(\boldsymbol{\theta}, \boldsymbol{J}_{\mathbf{1}}\right) \\
c_{\text {ineq } 2}\left(\boldsymbol{\theta}, \boldsymbol{J}_{\mathbf{2}}\right)
\end{array}\right] \\
& =\left[\begin{array}{c}
\sqrt{\left(\boldsymbol{T}_{\mathbf{2}}-\boldsymbol{J}_{\mathbf{1}}\right)^{T}\left(\boldsymbol{T}_{\mathbf{2}}-\boldsymbol{J}_{\mathbf{1}}\right)}-\left\|\overrightarrow{T_{2} J_{2}}\right\|-\left\|\overrightarrow{J_{2} J_{1}}\right\| \\
\sqrt{\left(\boldsymbol{T}_{\mathbf{2}}-\boldsymbol{T}_{\mathbf{1}}\right)^{T}\left(\boldsymbol{T}_{\mathbf{2}}-\boldsymbol{T}_{\mathbf{1}}\right)}-\left\|\overrightarrow{T_{2} J_{2}}\right\|-\left\|\overrightarrow{J_{2} T_{1}}\right\|
\end{array}\right] \leq \mathbf{0},
\end{aligned}
$$

where $\boldsymbol{c}_{\boldsymbol{i n e q}}\left(\boldsymbol{\theta}, \boldsymbol{J}_{1}, \boldsymbol{J}_{2}\right)$ is the matrix that consists of the inequality constraints of the target nodes which are identified by their positions on the body.

In summary, the constrained localization algorithm leads to find the minimum of constrained nonlinear multivariable function specified by:

$$
\begin{array}{r}
\hat{\boldsymbol{\theta}}=\arg \min _{\boldsymbol{\theta}} \sum_{i=1}^{4} \sum_{j=1}^{2}\left(\tilde{d}_{i j}-\sqrt{\left(\boldsymbol{R}_{\boldsymbol{i}}-\boldsymbol{T}_{\boldsymbol{j}}\right)^{T}\left(\boldsymbol{R}_{\boldsymbol{i}}-\boldsymbol{T}_{\boldsymbol{j}}\right)}\right)^{2} \\
\text { subject to }\left\{\begin{array}{c}
\boldsymbol{c}_{\boldsymbol{e} \boldsymbol{q}}\left(\boldsymbol{\theta}, \boldsymbol{J}_{\mathbf{1}}, \boldsymbol{J}_{\mathbf{2}}\right)=\mathbf{0} \\
\boldsymbol{c}_{\boldsymbol{i n e q} \boldsymbol{q}}\left(\boldsymbol{\theta}, \boldsymbol{J}_{\mathbf{1}}, \boldsymbol{J}_{\mathbf{2}}\right) \leq \mathbf{0} \\
\boldsymbol{J}_{\mathbf{2}}=\boldsymbol{J}_{\mathbf{1}}+\frac{\left\|\overrightarrow{J_{2} J_{1}}\right\|}{\left\|\overrightarrow{T_{1} J_{1}}\right\|}\left(\boldsymbol{T}_{\mathbf{1}}-\boldsymbol{J}_{\mathbf{1}}\right)
\end{array}\right.
\end{array}
$$

Remind that this optimization problem above is used to estimate the position of 2 target nodes (i.e. $\boldsymbol{\theta}=\left[\boldsymbol{T}_{\mathbf{1}}, \boldsymbol{T}_{\mathbf{2}}\right]^{T}$ ) on the left arm. For the target nodes on other limbs, the process is equivalent.

2) Cooperative ML Algorithm: In attempt to improve the precision as much as possible, the features of WSN are investigated and cooperative techniques seem favorable. In conventional localizations, the unknown-position nodes perform ranging measurements with the known-position nodes whereas cooperative ones enable the communications between unknown-position sensors [2][6]. As shown in Figure 3, the target (yellow) nodes on the arms make measurements with one another to introduce the information redundancies. Our study also considers the effect of the cooperative topology 


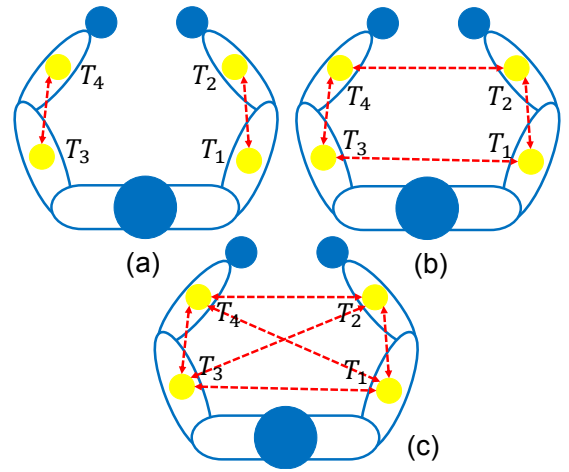

Figure 3. (a) Single-link cooperative scenario. (b) Quasi-mesh cooperative scenario. (c) Full-mesh cooperative scenario.

or spacial diversity in the quality of the localization. More particularly, three topologies i.e. single-link (SL), quasi-mesh (QM) and full-mesh (FM) are evaluated.

Firstly, considering the single configuration in Figure 3(a), only target nodes on the same arm are paired to perform the ranging measurements. Sensors on different arms are not allowed to exchange packets in this scenario. The solution for the ML estimation of the positions (of two sensors $T_{1}$ and $T_{2}$ ) in (7) becomes:

$$
\hat{\boldsymbol{\theta}}=\arg \min _{\boldsymbol{\theta}}\left[u(\boldsymbol{\theta}, \tilde{\boldsymbol{d}})+v\left(\boldsymbol{\theta}, \tilde{\boldsymbol{d}}^{\boldsymbol{c}}\right)\right],
$$

with

$$
u(\boldsymbol{\theta}, \tilde{\boldsymbol{d}})=\sum_{i=1}^{4} \sum_{j=1}^{2}\left(\tilde{d}_{i j}-\sqrt{\left(\boldsymbol{R}_{\boldsymbol{i}}-\boldsymbol{T}_{\boldsymbol{j}}\right)^{T}\left(\boldsymbol{R}_{\boldsymbol{i}}-\boldsymbol{T}_{\boldsymbol{j}}\right)}\right)^{2}
$$

and

$$
v\left(\boldsymbol{\theta}, \tilde{\boldsymbol{d}}^{\boldsymbol{c}}\right)=\left(\tilde{d}_{12}^{c}-\sqrt{\left(\boldsymbol{T}_{\mathbf{1}}-\boldsymbol{T}_{\mathbf{2}}\right)^{T}\left(\boldsymbol{T}_{\mathbf{1}}-\boldsymbol{T}_{\mathbf{2}}\right)}\right)^{2},
$$

where $\tilde{d}_{i j}^{c}$ denotes the cooperative measurement between the target node $i$ and the target node $j$. It can be seen that the likelihood function which is enclosed within square brackets in (12) has a new coefficient i.e. $v\left(\boldsymbol{\theta}, \tilde{\boldsymbol{d}}^{c}\right)$ in comparison with (7). This new coefficient expresses the cooperative localization between unknown nodes (i.e. $T_{1}$ and $T_{2}$ ) and provides the ML estimator with more information.

Next, the spacial diversity is fully exploited by quasi-mesh configuration in Figure 3(b) and full-mesh one in Figure 3(c). In these scenarios, the 4 sensors (i.e. $T_{1}, T_{2}, T_{3}$, and $T_{4}$ ) on the two arms communicate with one another to form cooperative mesh networks. Consequently, the solution for the ML estimation has the similar form as (12) apart from the cooperative coefficient which becomes:

$$
v\left(\boldsymbol{\theta}, \tilde{\boldsymbol{d}}^{\boldsymbol{c}}\right)=\sum_{(i, j) \in \mathcal{T}}\left(\tilde{d}_{i j}^{c}-\sqrt{\left(\boldsymbol{T}_{\boldsymbol{i}}-\boldsymbol{T}_{\boldsymbol{j}}\right)^{T}\left(\boldsymbol{T}_{\boldsymbol{i}}-\boldsymbol{T}_{\boldsymbol{j}}\right)}\right)^{2},
$$

where the estimated vector $\boldsymbol{\theta}=\left[\boldsymbol{T}_{\mathbf{1}}, \boldsymbol{T}_{\mathbf{2}}, \boldsymbol{T}_{\mathbf{3}}, \boldsymbol{T}_{\mathbf{4}}\right]^{T}$ and $\mathcal{T}$ is the set of cooperative pairs. In the case of the quasi-mesh topology,
$\mathcal{T}=\{(1,2),(1,3),(2,4),(3,4)\}$, while in the case of fullmesh one, $\mathcal{T}=\{(1,2),(1,3),(1,4),(2,3),(2,4),(3,4)\}$.

Concerning the cooperative approaches, the more cooperative measurements, the more information the ML estimator has. However, it is critical to consider the trade-off between extra information and its cost (e.g. over-the-air traffic, power consumption, computational load...). As a result, the cooperative techniques should give enough extra information but not largely redundant in order not to increase the complexity of the system. It explains why our cooperative sensor networks contain a maximum of four nodes. With these configurations, the most complex ML estimation is limited to a 12-dimensional optimization problem (each target node has three unknown coordinates on the $x$-axis, $y$-axis, and $z$-axis in Cartesian system).

3) Cooperative-cum-Constrained ML Algorithm: In the previous section, both constrained and cooperative localizations have been introduced. Each technique has a complementary strength to the other one. Thus, it is reasonable to merge both algorithms into one to fully exploit the body constraints, the spatial diversity and the measurement redundancies from the BAN in order to achieve superior accuracy. We call this as the Cooperative-cum-Constrained Maximum Likelihood (CCML) algorithm.

\section{Simulations And Results}

\section{A. Scenario Description and Simulation Parameters}

In this section, the framework to evaluate our proposed algorithms is presented. Regarding the BAN model, we developed a biomechanical model of a 1.8-meter body as depicted in Figure 4. Four reference nodes are attached on the chest, the back, the left hip, and the right hip to form the LCS whose origin is located at the stomach. Without loss of generality, the two nodes on the left arm are analyzed i.e. node $T_{1}$ between the shoulder and the elbow and node $T_{2}$ between the elbow and the wrist. In our evaluation framework, the performance of two conventional algorithms (the linear Least Square Error (LSE) and the ML), the family of cooperative techniques (CoopML) with the three different topologies, the constrained scheme (ConML), and the hybrid approaches (i.e. CCML) are evaluated by the Root Mean Square Error (RMSE) and the Geometric Dilution of Precision (GDOP) [1][9] as follows:

$$
\begin{aligned}
& \operatorname{RMSE}(\hat{\boldsymbol{\theta}}) \triangleq \sqrt{E\left\{\|\hat{\boldsymbol{\theta}}-\boldsymbol{\theta}\|^{2}\right\}}, \\
& \operatorname{GDOP}(\hat{\boldsymbol{\theta}})=\frac{\sqrt{\sigma_{x}^{2}+\sigma_{y}^{2}+\sigma_{z}^{2}}}{\sigma_{\text {range }}},
\end{aligned}
$$

where $\hat{\boldsymbol{\theta}}$ is the estimator of the target or true value $\boldsymbol{\theta}=$ $\left[T_{i}^{(x)}, T_{i}^{(y)}, T_{i}^{(z)}\right]^{T}$. $\sigma_{\text {range }}$ denotes the ranging deviation. $\sigma_{x}^{2}$, $\sigma_{y}^{2}$, and $\sigma_{z}^{2}$ represent the mean square errors for the $x$-axis, $y$-axis, and $z$-axis estimates, respectively. The ranging error is Gaussian noise with $\sigma_{i j}=\sigma_{\text {range }}=5 \mathrm{~cm}$. Therefore, independent realizations of ranging measurements are produced to conduct 10,000 simulations of each algorithm. 


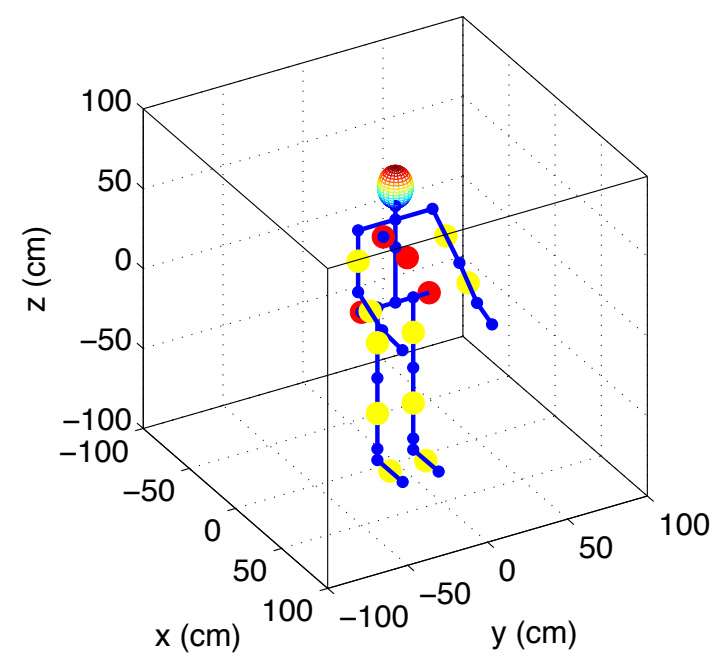

Figure 4. Sensor deployment in the biomechanical model for relative localization. The reference (red) nodes are attached on the torso to form the LCS while the target (yellow) nodes are mounted on the limbs.

\section{B. Performance Results by Simulations}

Figure 5 gives the information about the performance (through RMSE and GDOP) of the analyzed localization algorithms for the two nodes $T_{1}$ and $T_{2}$. The simplest technique (i.e. LSE) results in the greatest error due to the use of the linear estimator. Next, an improvement in accuracy is provided by the standard non-linear LSE (i.e. the ML) as a result of the consideration of the statistics of noise sources. Then, cooperative scenarios (i.e. CoopML-SL, CoopML-QM and CoopML-FM) reduce the inaccuracy gradually. The more complicated the cooperative network is or the more information redundancies we provide, the greater accuracy we achieve. A closer look at this figure reveals that the constrained technique (i.e. ConML) has different effects on the different target node's position estimates. Specifically, the decrease in the GDOP yielded by the body constraints of the $T_{1}$ 's position estimate is distinctly superior than that of the $T_{2}$. Furthermore, in case of the node $T_{2}$, there exists a slight rise in the RMSE. This is due to the fact that the position of the node $T_{2}$ (between the elbow and the wrist) has more degree of freedom than the node $T_{1}$ (between the shoulder and the elbow). Additionally, the body constraint related to the node $T_{2}$ depends on the $T_{1}$ 's position estimate. For this reason, an error in $T_{1}$ 's position can be magnified and would eventually ruin the $T_{2}$ 's position estimate. Lastly, the family of hybrid algorithms (i.e. CCMLSL, CCML-QM and CCML-FM) inherits the improvement in accuracy of both constrained and cooperative techniques.

Our results can be compared with other existing works using constrained localization in [4][5]. In the one hand, the constrained ML in [4] results in a relative drop in average RMSE per node of $36.2 \%-57.3 \%$ (depending on the node's position) compared with the standard ML but this work is limited to 2D positioning. On the other hand, this enhancement is only maximum $17 \%$ in [5] on account of the $3 \mathrm{D}$ localization

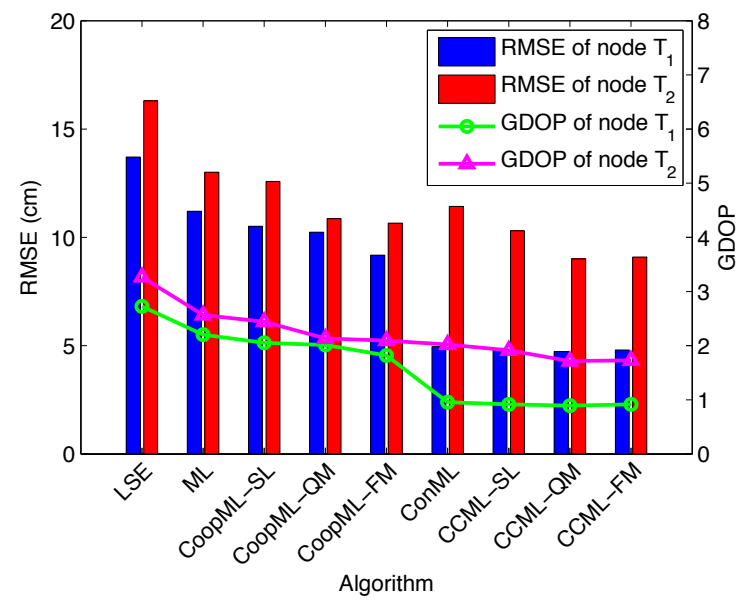

Figure 5. Relative localization RMSE and GDOP in case of $\sigma_{\text {range }}=5 \mathrm{~cm}$.

and the reduction in the number of constraints. In contrast, our constrained ML produces the relative enhancement of $32.4 \%$ over the ML. Moreover, our hybrid CCML can give more performance (maximum relative improvement of $42.6 \%$ ). In other words, our CCML algorithm can achieve the minimum absolute RMSE of $4.8 \mathrm{~cm}$ in $T_{1}$ 's position estimate and $9.09 \mathrm{~cm}$ in $T_{2}$ 's on condition of $5 \mathrm{~cm}$ ranging deviation.

\section{EXPERIMENTS}

This section introduces the real experiments that generate the real measurements in order to validate our algorithms.

\section{A. Experimental Setup}

The experiments are conducted using DecaWave's IR-UWB platforms [10] as shown in Figure 6 which have a bandwidth of about $900 \mathrm{MHz}$ centered at $6.5 \mathrm{GHz}$. Since the ToA is estimated, an antenna delay calibration is required to compensate for the system delays between physical timestamp and the signal presence at the antenna. The calibration is performed by placing a couple of DecaWave's platforms at different distances and adjusting the antenna delay until the range reading given by the device is correct. One platform is fixed, while the other is moved by $10 \mathrm{~cm}$ increment toward the static one from a distance of $200 \mathrm{~cm}$ to $10 \mathrm{~cm}$. At each separated distance, the calibration is conducted by fine-tuning the antenna delay to get at least 100 fine ranging measurements whose mean is close to the true value (i.e. approximately $1 \mathrm{~cm}$ of bias). Two typical configuration modes are used in our experiments i.e. mode 1 employs a low data rate of $110 \mathrm{kbps}$ and long preamble code of 1024 symbols whereas mode 2 uses a high data rate of up to $6.8 \mathrm{Mbps}$ and short code of 128 symbols to save energy [10]. Until the platforms is fine calibrated, these can be used to produce real measurements as input of our analyzed algorithms.

\section{B. Performance Results by Experiments}

Firstly, the relation between the antenna delay and the range is exposed in Figure 7. The calibrated delay not only depends on the platform setup but also on the distance. Alternatively, 


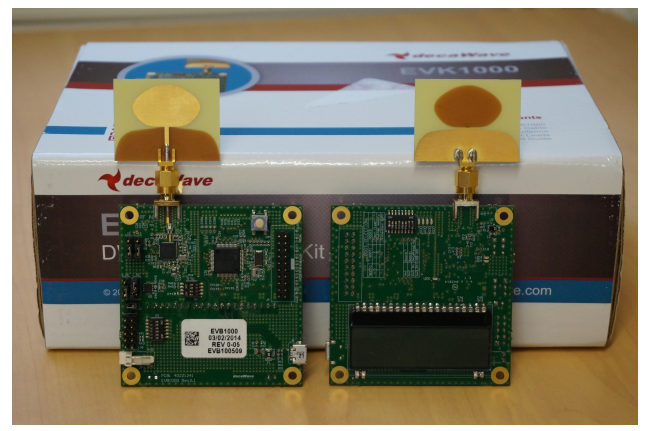

Figure 6. DecaWave's IR-UWB EVK1000 kit with UWB antennas.

the first mode has a more stable antenna delay than the second. Thus, the ranging performance of the mode 1 can guarantee higher accuracy in a wide variety of distances while the second can only ensure this accuracy locally in a limited range of distances (i.e. recalibration is required). This is due to the fact that a longer preamble code of mode 1 produces improved range performance and better ToA information [10]. In practice, the same antenna delay is calibrated on all UWB sensors to perform the ranging measurements in every distance. Thus, the mode 1 and an antenna delay of 514.22 ns are adopted to generate real ranging measurements.

Finally, we evaluate our proposed algorithms given the real measurements using DecaWave's platforms placed on the human body as in Figure 4. For each pair of nodes, 100 ranging measurements are performed to get an average behavior of the algorithms. Their performance are exposed in Figure 8. Expectedly, the body constraints and cooperation between nodes yield improvements in accuracy, which have been observed in the simulations. The order of the performance of the analyzed algorithms coincides with the simulation results. Expectedly, the best accurate position estimate is given by the CCML-FM. Particularly, the RMSE of $2.55 \mathrm{~cm}$ and GDOP of 1.038 are recorded in the node $T_{1}$ 's position estimate whereas in the node $T_{2}$ 's, the performance are degraded to $3.63 \mathrm{~cm}$ in RMSE and 1.341 in GDOP due to high degree of freedom position leading to loose body constraints as discussed.

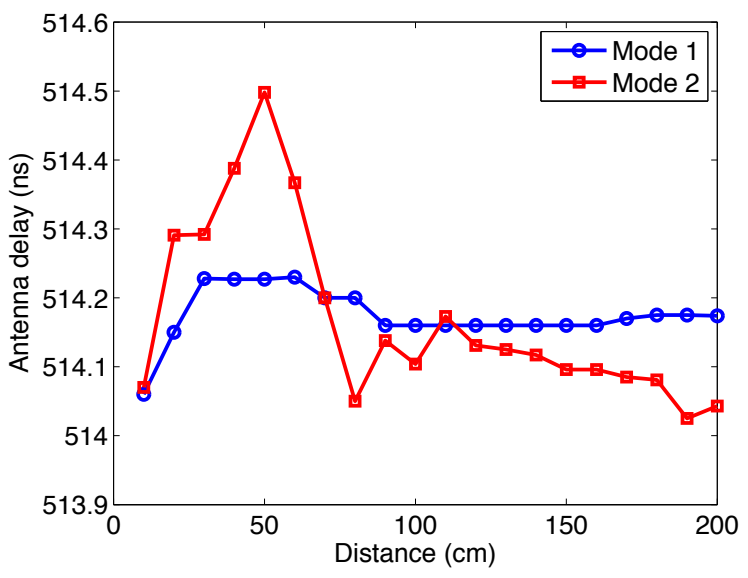

Figure 7. Calibrated antenna delay for different distances.

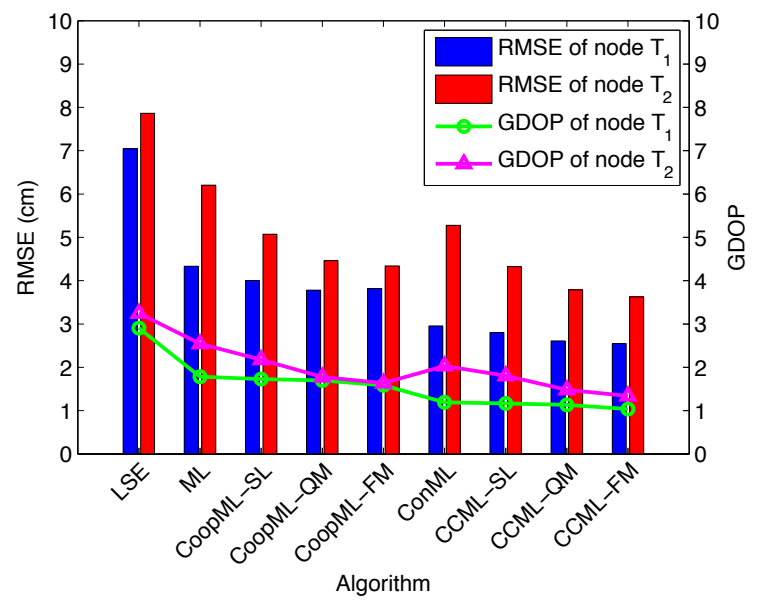

Figure 8. Relative localization RMSE and GDOP using real measurements.

\section{CONCLUSION}

In this paper, we have studied the localization in the context of wireless BAN. By fully exploiting the body constraints and/or cooperative communications, novel algorithms have been presented to increase accuracy performance. These proposed algorithms not only outperform various conventional unconstrained and/or non-cooperative techniques (relative decline in average RMSE and GDOP per node up to $54 \%$ and $56 \%$ respectively) but also leave behind other existing works on constrained localization in terms of accuracy and feasibility (our relative improvement of $43 \%$ against that of $17 \%$ in the work of Hamie et al [5] when considering the relative decrease in average RMSE per node with reference to the standard ML). Lastly, experimental setup shows an accuracy of from $2.55 \mathrm{~cm}$ to $3.66 \mathrm{~cm}$ depending on the nodes.

\section{REFERENCES}

[1] Z. Sahinoglu, S. Gezici, and I. Guvenc, Ultra-wideband Positioning Systems: Theoretical Limits, Ranging Algorithms, and Protocols. Cambridge University Press, 2008.

[2] H. Wymeersch, J. Lien, and M. Win, "Cooperative localization in wireless networks," Proceedings of the IEEE, vol. 97, pp. 427-450, Feb 2009.

[3] S. Gezici, Z. Tian, G. Giannakis, H. Kobayashi, A. Molisch, H. Poor, and Z. Sahinoglu, "Localization via ultra-wideband radios: a look at positioning aspects for future sensor networks," IEEE Signal Processing Magazine, vol. 22, pp. 70-84, July 2005.

[4] Z. Mekonnen, E. Slottke, H. Luecken, C. Steiner, and A. Wittneben, "Constrained maximum likelihood positioning for uwb based human motion tracking," in International Conference on Indoor Positioning and Indoor Navigation (IPIN'10), pp. 1-10, Sept 2010.

[5] J. Hamie, B. Denis, and C. Richard, "Constrained decentralized algorithm for the relative localization of wearable wireless sensor nodes," in IEEE Sensors 2012, pp. 1-4, Oct 2012.

[6] N. Patwari, J. Ash, S. Kyperountas, A. Hero, R. Moses, and N. Correal, "Locating the nodes: cooperative localization in wireless sensor networks," IEEE Signal Processing Magazine, vol. 22, pp. 54-69, July 2005.

[7] http://www.xsens.com/.

[8] J. C. Lagarias, J. A. Reeds, M. H. Wright, and P. E. Wright, "Convergence properties of the nelder-mead simplex method in low dimensions," SIAM J. on Optimization, vol. 9, pp. 112-147, May 1998.

[9] J. Zhu, "Calculation of geometric dilution of precision," Aerospace and Electronic Systems, IEEE Transactions on, vol. 28, pp. 893-895, Jul 1992.

[10] DecaWave Ltd., DW1000 User Manual: How to use, configure and program the DW1000 UWB tranceiver, 2013. 